Journal of Computer Science 4 (3): 239-244, 2008

ISSN 1549-3636

(C) 2008 Science Publications

\title{
Performance Study of Power Control Radio Resource Management Over WLANs
}

\author{
Z. I. Dafalla and M. B. R. Murthy \\ Multimedia University, Faculty of Engineering and Technology, \\ Jalan Ayer Keroh Lama, 75450 Bukit Beruang, Melaka, Malaysia
}

\begin{abstract}
Wireless Local Area Networks (WLANs) based on IEEE 802.11 standard have been rapidly growing. With limitations of the WLAN standard and rapid increase in wireless application demand, the air interface acts as bottleneck even in high-speed WLAN standards such as IEEE 802.11a and $802.11 \mathrm{~g}$ with expected data transmission rates of up to $54 \mathrm{Mbps}$. To improve the overall performance of IEEE 802.11 WLAN under large deployment and heavy application demand environment, Radio Resource Management (RRM) algorithms based on power control have been investigated and tested through simulation. The results show that controlling the Wireless Terminal's (WT) transmitter power to an optimum power level helps in increasing data throughput in WLANs and when the transmitter power level of a WT is increased beyond the optimum power level, the overall data throughputs drop drastically no matter how high the WT's transmitter power may be increased.
\end{abstract}

Key words: WLAN, radio resource management, IEEE 802.11

\section{INTRODUCTION}

IEEE 802.11 based WLAN systems have been widely deployed in many places in the present days. They offer mobility and are especially appropriate for temporary configurations like conference centres, for use in public areas such as an airport, coffee bar (these places are also called hot spots). The original 802.11 standard specifies a common Medium Access Control layer (MAC) and three Physical layers (PHYs) ${ }^{[1]}$. Two of these PHYs facilitate communications in the $2.4 \mathrm{GHz}$ Industrial Scientific and Medical (ISM) bands using Direct Sequence (DS) and Frequency Hopped (FH) Spread Spectrum techniques.

The third PHY facilitates communication over infra-red links. Data rates of up to $2 \mathrm{Mbits} / \mathrm{s}$ are facilitated by each of the PHYs. The 802.11 standard has subsequently been expanded considerably with several PHY extensions that can support data rates of up to $54 \mathrm{Mbits} / \mathrm{s}$. In a multi-access WLAN system, the MAC layer is the main element for determining the efficiency in sharing the communication bandwidth of the wireless channel. The MAC has two multi-access mechanisms: basic Distribute Coordination Function (DCF) and additional Point Coordination Function (PCF). The DCF is mandatory and most commonly used for 802.11 devices. It is based on a Carrier Sense Multiple Access with Collision Avoidance
(CSMA/CA), which imitates the mechanism used in Ethernet system and supports best effort delivery of packets and allows multiple users to fairly share a common communication channel.

Every 802.11 station that wants to transmit a frame will first sense the air channel, if it is busy, the station must wait for a random back off time and try again. As all stations in an 802.11 WLAN implement the same carrier sense and back off mechanism, all stations fairly share the communication channel statistically for a long period of time. For public deployment of 802.11 WLAN systems in large areas, multiple Access Points (APs) are used and are geographically distributed in an overlapping manner (similar to cellular systems). Each AP normally coordinates one air channel and covers a certain area offering all Wireless LAN Terminals (WT) accessing the AP. With more WTs gathering around the same AP and heavier application demand (such as downloading), these WLAN users will experience lower data throughput and longer delay due to congestions that occur in the air channel.

One way of expanding the WLAN system capacity is to mount more APs in the heavily congested area, but this method is an expensive approach because of limited available air channels. Accurate site surveys are needed in order to minimize interference between APs, and more capital investment for providing more APs. Moreover this approach cannot cope with a dynamic

Corresponding Author: $\quad$ Z. I. Dafalla, Multimedia University, Faculty of Engineering and Technology, Jalan Ayer Keroh Lama, 75450 Bukit Beruang, Melaka, Malaysia 
environment when the geographically distributed congestion varies with time (this usually happens in public area such as in an airport where WLAN users tend to gather into a certain area to wait for a flight and thus forming a temporary hot spot).

Performance of 802.11 wlan: The MAC specification in 802.11 standard families has remained largely unchanged but several new PHY layer specifications have been added ${ }^{[2]}$.

- $\quad$ The 802.11b PHY facilitates data rates of up to 11 Mbits/s, employing Complementary Code Keying (CCK) and Direct Sequence Spread Spectrum (DSSS), also in the $2.4 \mathrm{GHz}$ ISM band.

- The 802.11a PHY facilitates link adaptive data rates of up to $54 \mathrm{Mbits} / \mathrm{s}$, employing Coded Orthogonal Frequency Division Multiplexing Modulation (COFDM) in the $5 \mathrm{GHz}$ Unlicensed National Information Infrastructure (UN-II) band.

- The recent $802.11 \mathrm{~g}$ PHY specification is a high rate extension of $802.11 \mathrm{~b}$. The new PHY was based upon the link adaptive COFDM modulation scheme (maximum data rate of 54 Mbits/s) of 802.11a with mandatory backward compatibility with 802.11b. As distance from the AP increases, 802.11 based products provide reduced data rates to maintain connectivity.

The $802.11 \mathrm{~g}$ standard has the same propagation characteristic as $802.11 \mathrm{~b}$, because it transmits in the identical $2.4 \mathrm{GHz}$ band. Because the $5 \mathrm{GHz}$ radio signals do not propagate as well as the $2.4 \mathrm{GHz}$ radio signals, the 802.11a product range is limited compared to the $802.11 \mathrm{~b}$ or $802.11 \mathrm{~g}$ product range. Throughput is not the same as data rate for networking systems, because of overhead, environment and network compositions. The maximum throughput of $802.11 \mathrm{~b}$ is only $47 \%$ of $11 \mathrm{Mbits} / \mathrm{s}$. For $802.11 \mathrm{a}$ and $802.11 \mathrm{~b}$ a net throughput of $55 \%$ of $54 \mathrm{Mbits} / \mathrm{s}$ has been observed ${ }^{[3]}$.

Radio resource management in cdma systems: Code Division Multiple Access (CDMA) assigns unique codes to each communication to differentiate it from others in the same spectrum. By correlating the received signal with the same sequence, the desired signal can be demodulated. All unwanted signals will not be demodulated and will remain effectively as noise ${ }^{[4,5,6]}$. The system capacity is maximized if each mobile transmitter power level is controlled so that its signal arrives at the cell site with the minimum required Signal-to-Interference Ratio (SIR). This is because in CDMA, other users appear as interference to one user. Any excessive transmission power will increase the overall interference. If a mobile signal arrives at the access point with inadequate power or the signal is weak, this results into an unacceptable Bit Error Rate (BER) else the weak user will be dropped. If the received power from a mobile terminal is too large, the performance of this mobile terminal will be acceptable, but it will add undesired interference to all other users in the cell. In addition the mobile terminal has limited power supply hence it cannot transmit with excessive power for a long period of time.

Uplink power control is required for combating the near-far problem. The transmission path from mobile terminal closer to the access point is likely to have a much smaller attenuation factor than a user farther away. Thus both near and far mobile stations must adjust their transmission power in a way such that their signals arrive at the base station with equal received power levels. Downlink power control is required to increase the user capacity of the access point. Each access point has a limited transmission power budget that must be used to deliver signals to all the mobile terminals it serves ${ }^{[4,7,8]}$. Keeping the transmission power of each mobile terminal at lower level more mobile terminals can be accommodated.

Power Control: In CDMA systems, users create interference to each other to the extent of the correlation between their codes and the ratio of their received powers. Consequently, near-far effects in which strong users with higher received powers can overwhelm the communication quality of the week users with lower received powers must be mitigated. The system capacity is maximized if each mobile transmitter power level is controlled so that its signal arrives at the cell site with the minimum required Signal-to-Interference Ratio (SIR). Any excessive transmission power will increase the overall interference.

Open Loop Power Control: The function of open loop power control is to make the average power equal at each base station due to near-far and shadowing problems. Open loop power control does not have feedback loop and does not require resource overhead for signalling, it is relatively fast in controlling power especially when there is a sudden change in the channel condition. The transmitter estimates the channel condition (gain) and adjusts its transmission power accordingly. The estimation is based on the transmission on the other link, under the assumption that the uplink and downlink channel condition are closely correlated. 
Closed Loop Power Control: Closed loop power control is based on SIR measurement of RAKEcombined signals. It is used to minimize the transmission power according to the traffic load in both the reverse and forward links ${ }^{[5,9,10]}$. This results in increased capacity by reducing the interference to other users in the same cell and to other users in the other cells. Fig. 1 shows block diagram of closed loop power control system.

Closed loop power control comprises two types of loops: inner loop and outer loop.



Fig. 1: Block diagram of closed loop

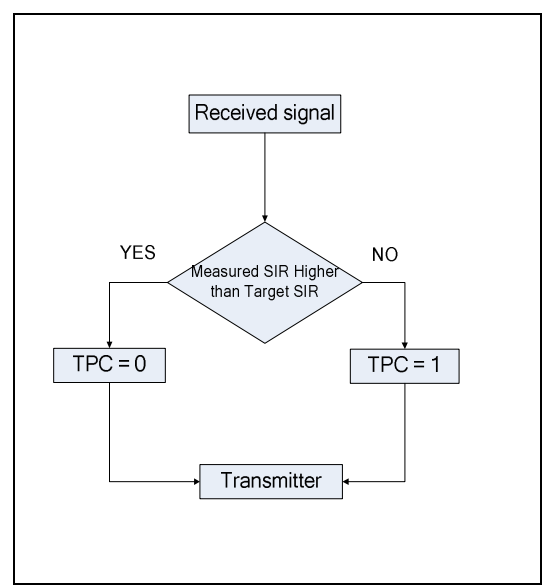

Fig. 2: Power control flow chart

Inner Loop Power Control: The receiver terminal calculates the received power level and determines if it is adequate according to design parameters. If necessary, it will request or order the transmitter to vary the transmit power in order to correct any deviation from the required level ${ }^{[5,11,12]}$. In this system, the receiver constantly measures the received signal level.
The measured level is compared to Signal-toInterference Ratio (SIR) target and a command/request message is fed back to the transmitter so that the target level can be reached and then maintained.

The access point estimates the SIR of the received uplink and it will generate Transmit Power Control (TPC) commands that are transmitted once per slot according to the following rule: if measured SIR is higher than target SIR, then the make TPC command to transmit a "0", while if the measured SIR is lower than target SIR, then make the TPC command to transmit a "1". Upon reception of one or more TPC commands in a slot, the mobile station derives a single TPC command for each slot, combining multiple TPC commands if more than one is received in a slot and power level is controlled as explained below. Fig. 2 shows the flow chart of Power Control. Two algorithms are supported by the mobile station for deriving a TPC command described below.

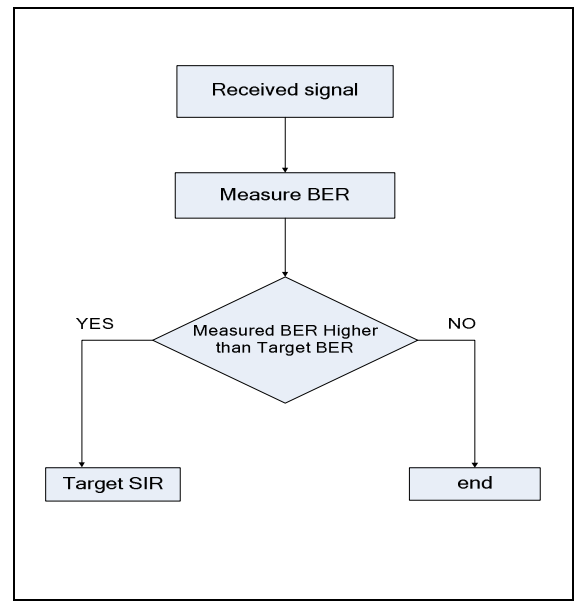

Fig. 3: Outer loop power control flow chart

Algorithm 1: In this algorithm the power control changes the mobile transmitter output power whenever a single TPC command is received.

Algorithm 2: If all five consecutive TPC commands received by a mobile station are estimated to be down or 0 the transmitter power of the mobile station is reduced by $1 \mathrm{~dB}$.

- If all five estimated TPC commands received by the mobile station are up or 1 the mobile station transmitter power is increased by $1 \mathrm{~dB}$.

Otherwise the mobile transmit power is not changed.

The power control algorithm determines which algorithm is to be used. 


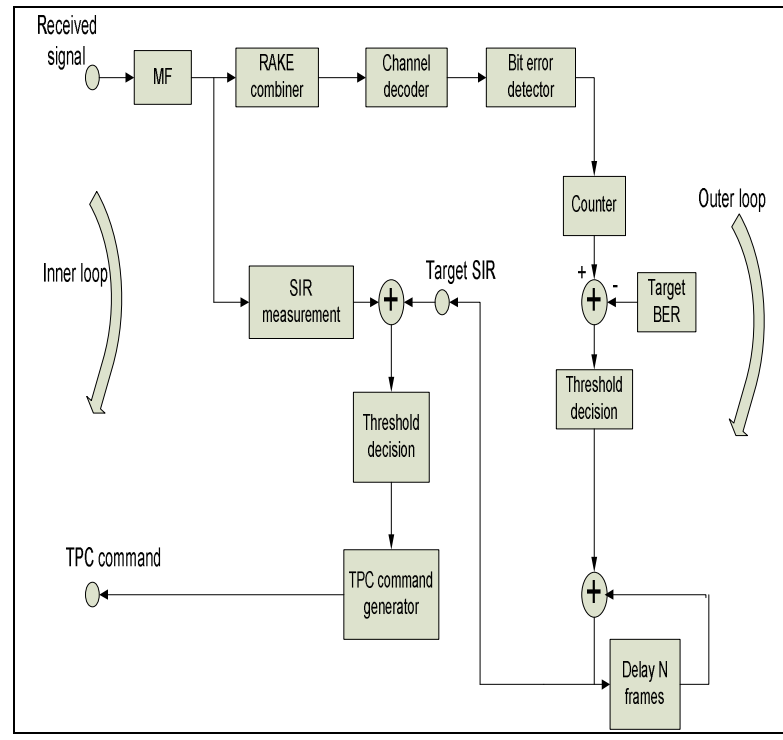

Fig. 4: Block diagram of inner loop and outer loop

Table 1:Topology parameters

\begin{tabular}{ll}
\hline Scenario Area & $62500 \mathrm{~m}^{2}$ \\
\hline Number of Access Points & 1 \\
Number of Wireless Nodes & 8 \\
Number of Wired Nodes & 10 \\
Application Type & HTTP, FTP \\
\hline
\end{tabular}

Outer loop power control: Outer loop power control is used to maintain the quality of communication at the level of bearer service quality requirement. Outer loop power control generates transmission power control command by comparing the measured power to target power. Flow chart of outer loop power control is shown in Fig. 3.

The uplink outer loop power control is responsible for setting a target BER for each individual uplink inner loop power control. This target BER is updated for each mobile station according to the estimated uplink quality and BER for each Radio Resource Control connection. The downlink outer loop power control helps the mobile terminal to converge to required link quality (BER) set for the wireless network.

Simulation design: In order to study the behaviour of Radio Resource Management (RRM) in WLANs through power control, a power monitor model was developed to enhance the access point node model existing in OPNET. The designed power monitor model is based on closed loop power control mechanism, which consists of inner loop and outer loop Fig. 4. The function of inner loop is to determine the power in $\mathrm{dB}$ allotted to a transmitter channel. The Bit Error Rate
(BER) is determined based on outer loop. The power monitor model compares the value of BER (in $\mathrm{dB}$ ) and the value of transmitter channel power (in $\mathrm{dB}$ ) to determine whether to increase or decrease the transmitter channel power (in $\mathrm{dB}$ ) of the mobile station. The increment or decrement is based on power control algorithm explained in the previous section of this study. The power monitor model then converts the new value of transmitter channel power in $(\mathrm{dB})$ to units of watts, after making comparison and going through the power control algorithm. The latest value of the transmitter channel power is then assigned to the mobile station transmitter.

Table 2: Simulation parameters

\begin{tabular}{ll}
\hline Simulation time & 1 hour \\
\hline Coverage radius & $100 \mathrm{~m}$ \\
Simulation Result & Delay(s), Throughput(bps) \\
Node that concern & User 1, User 8, Access Point \\
\hline
\end{tabular}

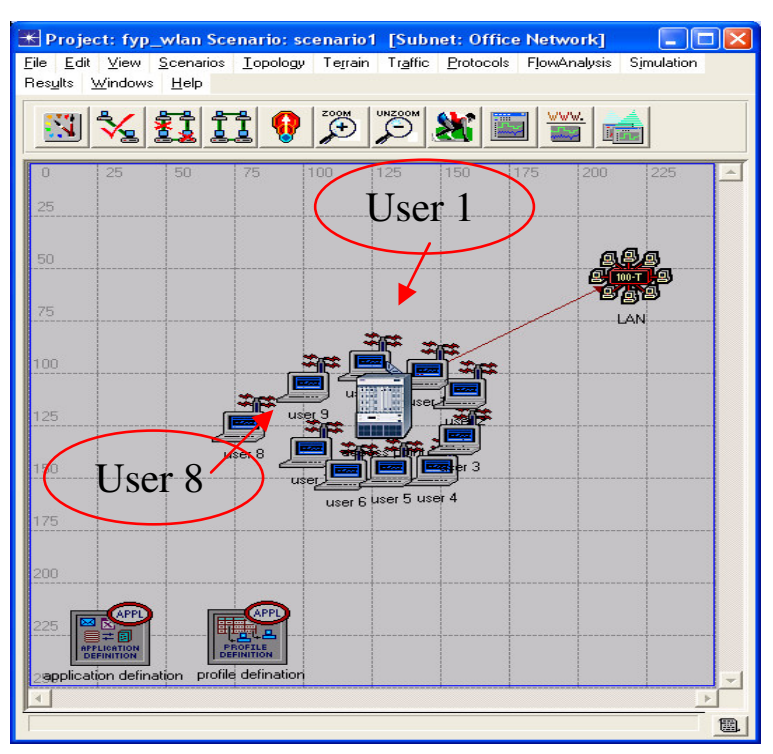

Fig. 5: WLAN Topology

Tables 1 and 2, summarize the WLAN topology configurations, and simulation parameters respectively. All wireless nodes were configured according to IEEE $802.11 \mathrm{~b}$ standard.

Fig. 5 shows a WLAN topology of an area of $62500 \mathrm{~m}^{2}$ designed in OPNET. The topology has eight wireless terminals (User 1-8) connected through wireless medium to one Access Point (AP). The access point is connected to Local Area Network (LAN), which consists of 10 users by using $100 \mathrm{Mbps}$ Unshielded Twisted Pair (100BASET) backbone. 


\section{RESULTS AND DISCUSSION}

The performance of User 1 represents an example scenario similar to users (Wireless Terminals) close to the access point and User 8 represents a wireless terminal farther away from the access point. The coordinates of the access point, User 1 and User 8 and distances between the access point and User 1 and User 8 are shown in Table 4.

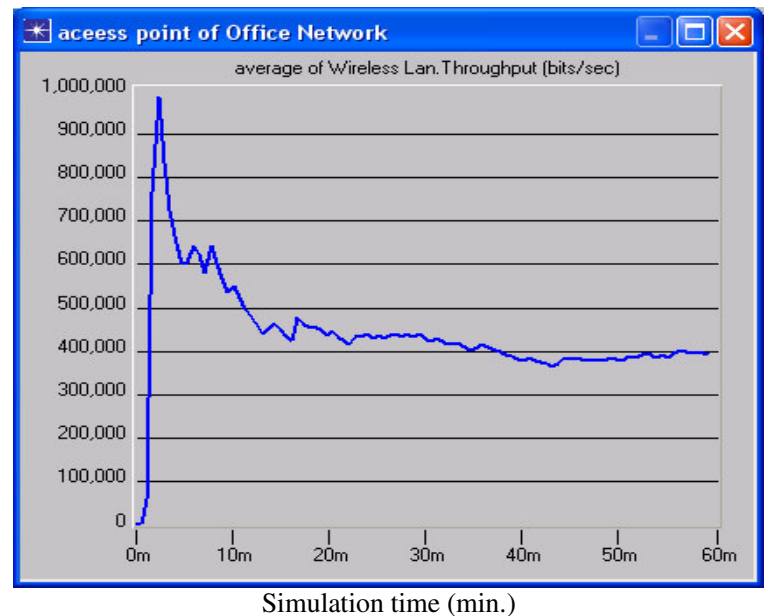

Fig. 6: User1 transmitter power $=0.01 \mathrm{~W}$

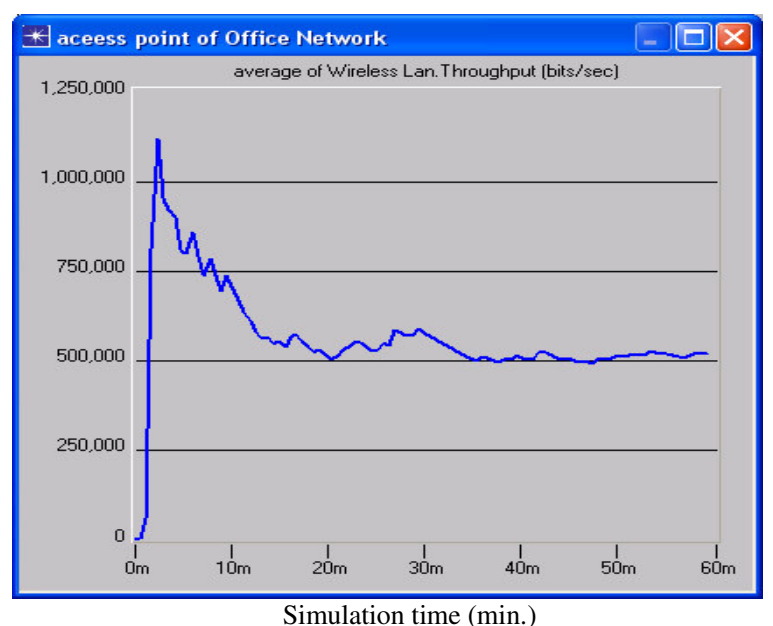

Fig. 7: User1 transmitter power $=0.03 \mathrm{~W}$

Figures 6, 7, and 8 show the case where the access point transmitter power was made constant at $0.01 \mathrm{~W}$ while the wireless terminal 1 (user 1) transmit power was gradually increased. As can be observed in Fig. 6, the average throughput against simulation time for the access point in bits per second is around $400 \mathrm{kbps}$ when the user transmit power was set to $0.01 \mathrm{~W}$. In Fig. 7 when user 1 (WT 1) transmitter power was increased to $0.03 \mathrm{~W}$, the average throughput at the access point increased to $500 \mathrm{kbps}$. In Fig. 8 when users 1 transmit power was increased to $0.05 \mathrm{~W}$, the average throughput at the access point dropped to $400 \mathrm{kbps}$.

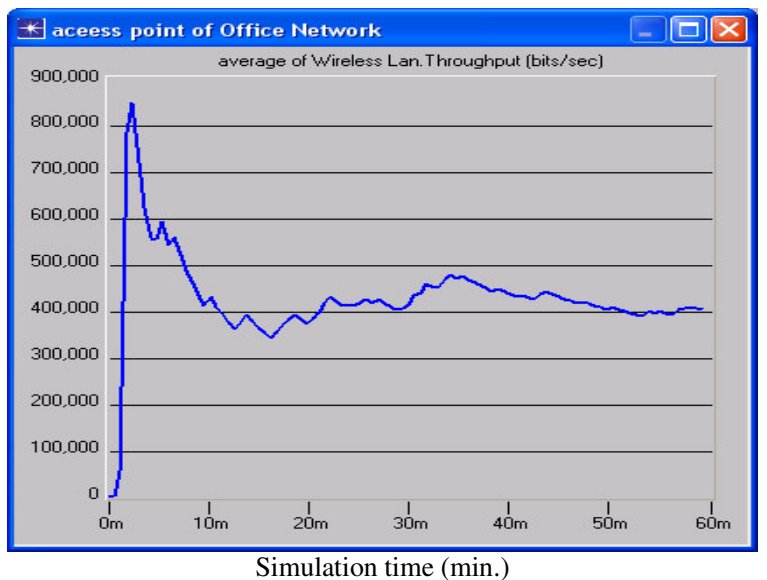

Fig. 8: User1 transmitter power $=0.05 \mathrm{~W}$

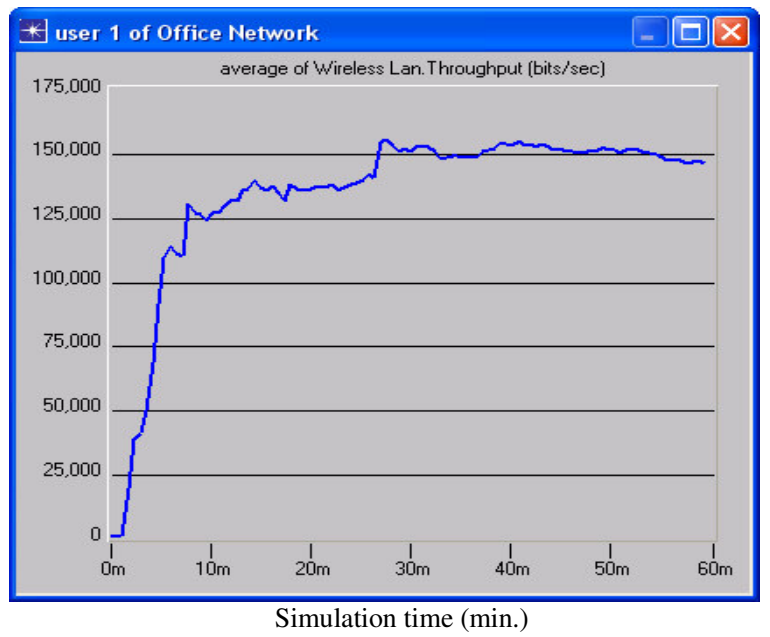

Fig. 9: User 1 transmitter power $=0.01 \mathrm{~W}$

Figures 9, 10 and 11 show the average throughputs in bits per second for User 1 when its transmitter power was configured at $0.01 \mathrm{~W}, 0.03 \mathrm{~W}$ and $0.05 \mathrm{~W}$ respectively with access point power fixed at $0.01 \mathrm{~W}$. The average throughput for User 1 (WT1) increased from $150-220 \mathrm{kbps}$ when the transmitter power was changed from $0.01 \mathrm{~W}$ and $0.03 \mathrm{~W}$ respectively (Fig 9 and 10). The increase in the average throughput is due to increment in the user 1's transmitter power from $0.01 \mathrm{~W}-0.03 \mathrm{~W}$. However the average throughput decreased from $220 \mathrm{kbps}$ to $170 \mathrm{kbps}$ when user 1's transmitter power was increased to $0.05 \mathrm{~W}$ (Fig. 11). This is in contrast to previous analysis. 


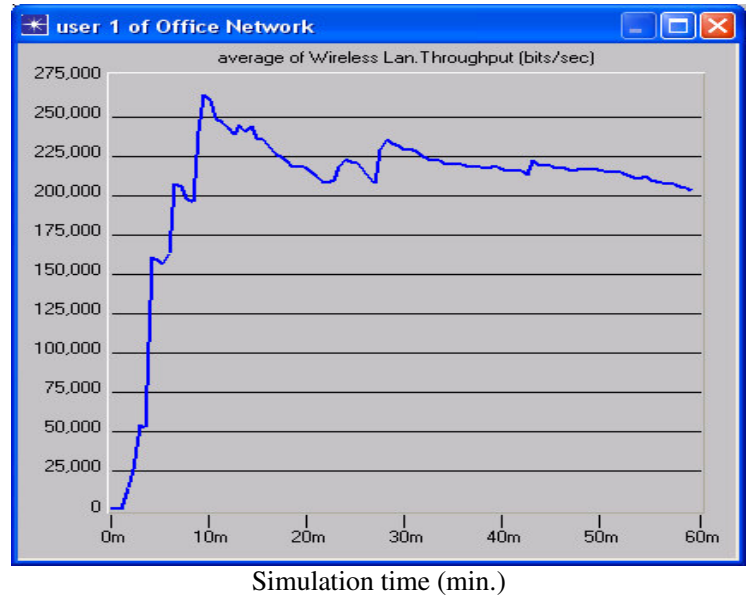

Fig. 10: User 1 Transmitter power $=0.03 \mathrm{~W}$



Fig. 11: User 1 Transmitter power $=0.05 \mathrm{~W}$

The major negative effect of excessive transmission power is that it increases the overall interferences in the wireless network. In 3G systems and beyond where CDMA is the predominant access technique, wireless users create interference to each other that affect the correlation between their codes. Consequently, near-far effects in which strong WTs with higher power can overwhelm the communication quality of the week users with lower powers is observed. Even if so, excessive transmitter power from a particular WT will be translated into interference which affects the overall data throughput from the transmitting user as well. The system capacity is maximized if each WT's transmitter power level is controlled so that its signal arrives at the Access point with a minimum interference.

\section{CONCLUSION}

Power control is one the most important RRM techniques employed in a wireless network. It balances the received powers of the users so that no user should create excessive interference in the Wireless Local Area Network. In this paper we have shown the importance of power RRM strategies in designing a wireless network and this has been tested through simulation. The results show that optimizing power control algorithms in a wireless network is very crucial if higher throughput is to be achieved. Also important is the simplicity of these algorithms so that there should not be too much computational load on the network while executing the algorithms. As part of an on going work, we are currently studying the effect of power control on multimedia traffic that requires different QoS and hence different user transmission powers.

\section{REFERENCES}

1. Anna Hac, 2003. Mobile Telecommunications Protocols for Data Networks. John Wiley and Sons, Inc.

2. IEEE 802.11 WG, Aug., 1999. Part 11: Wireless LAN Medium Access Control (MAC) and Physical Layer (PHY) specifications Standard.

3. The New Main Stream of WLAN Standard, White Paper, Broadcom, July 2003.

4. Kamerman. A. G. Aben, Sept., 2000. Throughput Performance of Wireless LANs Operating at 2.4 and $5 \mathrm{GHz}$, Personal, Indoor and Mobile Radio Communications. Sept., 2000. The 11th IEEE International Symposium on, 1:18-22.

5. Bigham. L. Du, J. and L. Cuthbert, 2002. Intelligent Cellular Coverage Control According To Geographic Call Traffic Distribution, 3g Mobile Communication Technologies. Third International Conference on (Conf. Publ. No. 489).

6. Perkins. C. E, 1998. Mobile networking through mobile IP, Internet Computing, IEEE, 2: 58-69.

7. IST Project SHUFFLE, 2001. An agent based approach to controlling resources in UMTS networks. last visited on Jan. 2001.

8. Rappaport .S, 2000. Wireless Communications, Principles and Practice. $2^{\text {nd }}$ edition, Prentice Hall.

9. Gim Geier, 2003. Wi-Fi Planet. [last visted on 20 June 2005].

10. Gary S. R and E. John. 2000. An Introduction to Wireless Technology. Prentice Hall.

11. Jiangzhou W and Ng. Tung-Sang. 2002. Advances in 3G Enhanced Technologies for Wireless Communiactions. Artech House.

12. Riaz Esmailzadeh and Masao Nakagawa, 2003. TDD-CDMA for wireless communications., Artech House. 